most positive results occurred in the first three cultures. Over 14 months 498 blood samples were cultured from 295 patients; 49 cultures were positive but only 27 of these were considered significant; of the remainder 16 grew Staphylococcus albus and six other commensals.

The principal bacteria found in the cultures that were considered significantly positive were pneumococci in seven, Staph aureus in six, Streptococcus viridans in four, and "Proteus vulgaris" in three (this is an uncommon species, since most infections are due to $P$ mirabilis). Clinical diagnoses are separately listed and not related to the bacteriological diagnoses. Positive cultures were obtained in six out of 95 patients with respiratory infection, four out of 28 with suspected bacterial endocarditis, five out of 32 with "non-specific malaise," and in one or two with recent onset of confusion, uncontrolled diabetes, and miscellaneous septic states. The authors emphasise that clinical signs of septicaemia, including fever, shock, and oliguria, were often lacking. Over half the patients with significant bacteraemia died, with the important exception of those with pneumococcal infection, all of whom survived.

Several lessons may be learnt from these results. Firstly, bacterial endocarditis should be strongly suspected in any elderly patient with a heart murmur, even in the absence of all other signs. A second, even more important lesson is the value of blood culture in diagnosing pneumococcal lung infection, for only 22 of these 95 patients, including only one of the six with pneumococcal bacteraemia, had a positive result on sputum culture. As the authors admit, there may have been other reasons for this, such as a poor technique in collecting the sputum; and it must be admitted that poor cultural technique may also have been a factor. It would be interesting to know in how many of these 95 specimens intraperitoneal mouse inoculation would have provided the answer-and provided it much more rapidly than ordinary cultivation. Lastly, it is important to recognise that in the elderly such nondescript complaints as mere "malaise" may result from bacteraemia. This is clearly a field in which blood culture may be the only means of reaching a diagnosis.

\footnotetext{
1 Sonnenwirth, A C, ed, Bacteremia, Laboratory and Clinical Studies. Springfield, Thomas, 1973.
}

2 Denham, M J, and Goodwin, G S, Age and Ageing, 1977, 6, 85.

\section{Immunological deficiency and the risk of cancer}

We now know that a child with a primary immune deficiency disease has a hundredfold increase in the incidence of cancer over that in a population of normal children. Dr Robert Good of the Memorial Hospital, New York, announced at a symposium on lymphomas held in Paris this June that 227 (9\%) cases of malignant tumours had now developed among the 2500 patients known to have congenital immune deficiency diseases. This special group of immune deficiency diseases, lying as they do at the intersection of immunology and carcinogenesis research, may hold the key to several of the contemporary questions about the causes of cancer. The high risk of cancer in immune deficiency diseases presents researchers with the challenge of designing a study that will identify one of several factors that increases this risk. Meanwhile, laboratory-based scientists are conducting studies that may one day enable some form of biological engineering to correct the fundamental fault in the immune system or provide an acceptable healthy line of cells to compensate for those that are defective.

The distribution of tumour types in immune deficiency diseases does not follow the pattern we might expect if the risk of developing cancer was non-specifically increased in these children. Of the 151 tumours recorded in the immune deficiency diseases registry in $1973,{ }^{12}$ over half (88) were lymphoreticular tumours. This group of tumours were predominant in all the main types of immune deficiency diseaseisolated deficiency, Wiskott-Aldrich syndrome, ataxia telangiectasia, common variable hypogammaglobulinaemia, and severe combined immunodeficiency. In ataxia telangiectasia the risk of cancer is as high as $10 \%$. On the other hand, the incidence of tumours of the central nervous system, bones, and other sites such as the kidney is much lower than would be expected if the deficiency disease simply generally increased the risk of developing cancer. ${ }^{34}$ Leukaemias, for example, are only half as common as would be expected from their normal percentage contribution to the spectrum of children's tumours.

The distribution of subgroups of lymphoreticular cancers is also abnormal. Dr Good estimated that in an unselected population of such cancers $12 \%$ would be B-cell type, $18 \%$ T-cell type, and $70 \%$ classified as nul. In patients with immune deficiency diseases, however, $78 \%$ of these cancers were B-cell type and $22 \% \mathrm{~T}$-cell type, which again shows the special biological selection operating in these conditions.

Another view of the relation of immunodepression and cancer comes as an unwelcome consequence of replacing diseased organs by grafting, which necessitates chronic immunosuppression to prevent graft rejection. Penn ${ }^{5}$ has recently reviewed this phenomenon and calculated that the risk of cancer is increased 10 to 100 times by iatrogenic immunosuppression. In patients with such secondary immunodeficiency, epithelial tumours accounted for $67 \%$ of the cancers, with the lip, skin, and cervix being most often affected. Hoover and Fraumeni, ${ }^{6}$ reviewing 6297 patients given kidney transplants in 1951-71, calculated that their risk of developing lymphoma was 30-40 times the expected risk in age-matched controls.

We have no evidence that tissues from patients with immune deficiency diseases show an increased susceptibility to carcinogenic viruses, such as neoplastic transformation by SV40 virus, ${ }^{7}$ though fibroblasts from patients with Fanconi's syndrome $^{8}$ and Down's syndrome ${ }^{9}$ do show an increased susceptibility. Some workers suspect that chromosome 14 may be abnormal in patients with immune deficiency diseases but this needs further study. Indeed, hard evidence on how the basic defect of an immune deficiency syndrome or secondary immunosuppression is linked to the induction of cancer is still lacking. The preferential development of certain types of tumours weighs against a glib explanation that the cancers arise because of the lack of protection from an immunosurveillance system.

\footnotetext{
1 Kersey, J H, Spector, B D, and Good, R A, International fournal of Cancer, 1973, 12, 333.

${ }^{2}$ Kirkpatrick, C H, Birth Defects, Original Article Series, 1976, $12,61$.

${ }^{3}$ Kersey, J H, Spector, B D, and Good, R A, Fournal of Pediatrics, 1974, 84, 263.

${ }^{4}$ Miller, R W, Fournal of Pediatrics, 1969, 75, 685.

5 Penn, I, Cancer, 1974, 34 (suppl), 858.

6 Hoover, R, and Fraumeni, J F, Lancet, 1973 2, 55.

7 Kersey, J H, Gatti, R A, and Good, R A, Proceedings of the National Academy of Science, USA, 1972, 69, 980.

8 Todaro, G J, Green, H, and Swift, M R, Science, 1966, 153, 1252.

9 Todaro, G J, and Martin, G, Proceedings of the Society for Experimental Biology and Medicine, 1967, 124, 1232.
} 